\title{
Four-body treatment of the antihydrogen-positronium system: binding, structure, resonant states and collisions
}

\author{
Piotr Froelich ${ }^{1} \cdot$ Takuma Yamashita $^{2}$ - Yasushi Kino ${ }^{2}$. Svante Jonsell ${ }^{3}$. \\ Emiko Hiyama ${ }^{4} \cdot$ Konrad Piszczatowski $^{1}$
}

Published online: 6 May 2019

(C) The Author(s) 2019

\begin{abstract}
We have developed a coupled-rearrangement-channel method allowing the rigorous nonadiabatic treatment of the multi-channel scattering problem for four particles. We present the study of the binding, resonant and collisional properties of the $\bar{H}-P s$ system with the total angular momentum $J=0^{+}$(singlet positronic configuration). The binding energy, the lifetimes of the resonant states and the collisional cross sections are calculated and discussed. We present the preliminary cross sections for the elastic and inelastic $\bar{H}-P s$ scattering, notably for the excitation of $P S$ and for the rearrangement reaction producing the $\bar{H}^{+}$ions.
\end{abstract}

Keywords Antihydrogen · Positronium · Collisions

\section{Introduction}

The experiment aiming to study the Gravitational Behavior of Antihydrogen at Rest (GBAR) involves the step whereby the antihydrogen atoms collide with positronium atoms to form the positronium hydride molecular ions $\vec{H}^{+}$suitable for sympathetic cooling [1-4]. In view of that we have undertaken the investigation of the $\bar{H} P s$ system with a method capable to describe the multichannel aspects of the $\bar{H}-P s$ scattering, including the elastic and inelastic processes, particularly the rearrangement reaction leading to the $\vec{H}^{+}$production.

The $\bar{H} P$ s system has been studied before, both in works concentrating on its ground state [5-8] and in works treating the scattering [9-12]. In the present work we use the description that is suitable to study both these aspects. We study the system in terms of several sets of Jacobi coordinates that are natural for its sub-cluster structure; this facilitates analysis of

This article is part of the Topical Collection on Proceedings of the 13th International Conference on Low Energy Antiproton Physics (LEAP 2018) Paris, France, 12-16 March 2018

Edited by Paul Indelicato, Dirk van der Werf and Yves Sacquin

Piotr Froelich

piotr.froelich@kemi.uu.se

Extended author information available on the last page of the article. 
the binding energy, formation and decay of resonances and the treatment of multichannel scattering.

To describe the structure of the the $\bar{H}-P s$ system we apply the variational approach based on the Gaussian Expansion Method (GEM) [13, 14]. The variational manifold explicitly contains contributions from various rearrangement channels expressed in terms of the appropriate Jacobi coordinates. At the same time, the use of Jacobi coordinates allows an efficient and rigorous treatment of the scattering cross sections.

The binding energy is calculated and discussed in terms of the contributions from various rearrangement channels. Channel analysis helps to converge the binding energy and throws light on the structure and collisional properties of the system. The structure of the system is presented in terms of the correlation functions that portray the spatial distribution of the particles. This helps to understand the structure and dynamics of the system, in particular the change of the $\bar{H}^{+}$upon binding of an electron, and the coexistence of the atomic and molecular features of $\bar{H} P s$.

The resonant states and their life-times are calculated variationally using GEM in conjunction with the Complex Coordinate Method (CCM). The energies and widths of the resonances are analysed with respect to the channel composition of the resonant wave functions.

Based on the variational description of the four-body system, we apply the Coupled Rearrangement Channels Method (CRCM) to calculate the cross sections for $\bar{H}-P s$ collisions. The outer part of the total $\bar{H} P S$ wave function is made to satisfy the appropriate scattering boundary conditions for the collisional fragments. This is facilitated by the use of Jacobi coordinates in the description of the multi-channel structure of the wave function, that expressly contains various rearrangement channels. The scattering matrix $S$ and the cross sections are obtained from the coupled, non-local integro-differential equations that explicitly couple the collisional channels of interest.

\section{General outline of the method}

The scattering cross sections were calculated using the Coupled Rearrangement Channel Method $[15,16]$. In this method one constructs the total scattering wave function $\Psi$ in terms of the inner part (that describes the internal, highly correlated part of the 4-body system) and the outer part (that describes the asymptotic motion of the various possible scattering fragments), see (1).

The inner part is expanded in terms of the square-integrable, 4-body basis functions $\Phi_{v}$ which are taken to be the eigenfunctions of the matrix eigenvalue problem for the total Hamiltonian of the system. Their construction is described in the next section. The important aspect of our approach is the use of several Jacobi sets of coordinates in the expansion of one and the same total wave function, and each function $\Phi_{v}$ contains contributions from several functional manifolds expressed in different sets of Jacobi coordinates (corresponding to different arrangement channels, see Fig. 1). The manifolds corresponding to various sets of coordinates are different from each other, even if the basis functions that span these manifolds are similar (they are all gaussians). In other words, various sets of Jacobi coordinates describe different parts of the configuration space of the system. Consequently, the problem of linear dependencies is alleviated and we do not suffer from the overcompletness.

The outer part is given in terms of the (a priori unknown) channel functions $\chi_{c}\left(R_{c}\right)$ of the relative motion between various scattering fragments. The channel functions are coupled to each other by being matched to the inner part and becoming part of the total wave function $\Psi$. It is the asymptotic behavior of the channel functions that give the information about the 


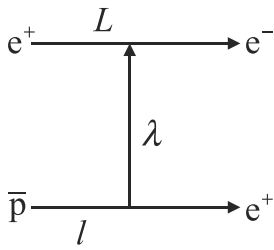

$c=13$

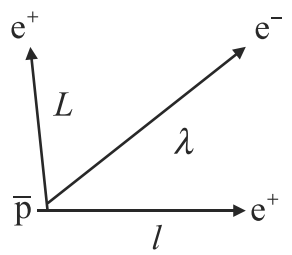

$c=1$

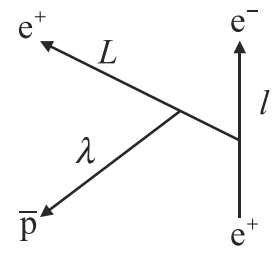

$c=10$

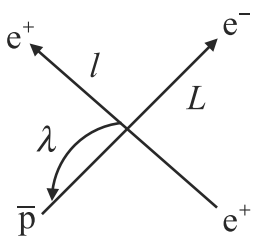

$c=15$

Fig. 1 Rearrangement channels for $\bar{H} P s$. We see the various arrangements of Jacobi coordinates, and the angular momenta for the wave functions in these coordinates. For the symmetrization purposes, we also include rearrangement channels with the positrons exchanged

scattering cross sections. To this end, the total scattering wave function for $\bar{H} P s$ is written as

$$
\begin{aligned}
\Psi= & \sum_{v} b_{v} \Phi_{v}+\sum_{c \in H} \phi_{c}^{(2)}\left(\mathbf{r}_{c}\right) \phi_{c}^{(2)}\left(\mathbf{q}_{c}\right) Y^{(c)}\left(\hat{R}_{c}\right) \frac{\chi_{c}\left(R_{c}\right)}{R_{c}} \\
& +\sum_{c \in K} \phi_{c}^{(3)}\left(\mathbf{r}_{c}, \mathbf{q}_{c}\right) Y^{(c)}\left(\hat{R}_{c}\right) \frac{\chi_{c}\left(R_{c}\right)}{R_{c}} .
\end{aligned}
$$

In the above, all 3 sums are over 4-body basis functions, but these functions are differently constructed. The first sum contains the variational solutions of the 4-body problem, obtained in the basis that contains functions from as many Jacobi arrangement channels as needed for the good description of the binding energy. In the present calculation we have used 7 Jacobi arrangement channels (out of 15 possible for the system) in the single calculation that couples all of them.

The remaining summations are over 4-body functions that describe the asymptotic character of those open scattering channels that one wishes to consider and couple. Here, function $\phi_{c}^{(n)}$ describes the $n$-body fragment of the scattering channel $c ; \mathbf{r}_{c}, \mathbf{q}_{c}$ and $\mathbf{R}_{c}$ are the 3 Jacobi coordinates in that channel.

The second sum is over channels that asymptotically contain two 2-body fragments. In the present case, these fragments are $\bar{H}$ and $P s$ and are described by functions $\phi_{c}^{(2)}\left(\mathbf{r}_{c}\right), \phi_{c}^{(2)}\left(\mathbf{q}_{c}\right)$, respectively. The relative motion of the centers of mass of these fragments is described by the function $\chi_{c}$ in terms of the third Jacobi coordinate $R_{c}$ that connects the mass centers. The three Jacobi coordinates for the channel functions in this group look graphically like the letter $H$ (see Fig. 1), we abbreviate the summation as $c \in H$.

The third sum is over channels that asymptotically contain a three-body fragment $\left(\bar{H}^{+}\right.$ or $\left.P s^{+}\right)$. The wave functions for these fragments $\left(\phi_{c}^{(3)}\left(\mathbf{r}_{c}, \mathbf{q}_{c}\right)\right)$ are obtained by separate 3body calculations. The motion of the 4 th particle is described by the function $\chi_{c}$ in terms of the third Jacobi coordinate $R_{c}$ that connects the lone particle to the CM of the 3-body fragment. The three Jacobi coordinates for the functions in this group look graphically like the letter K (see Fig. 1), we abbreviate the summation as $c \in K$.

As will be seen in the following, expansion coefficients $b_{v}$ and functions $\chi_{c}$ in (1) are determined simultaneously via the self-consistent, integro-differential procedure.

We first describe the variational procedure that generates the expansion functions $\Phi_{v}$ for the inner part of the scattering wave function. 


\section{Binding energy and structure of $\bar{H} P s$}

To obtain the inner part of the total wave function, we solve the Schrödinger equation for $\bar{H} P s$ by means of the variational approach, using the Gaussian Expansion Method in Jacobi coordinates $[13,14]$. The expansion functions $\Phi_{v}$ are taken to be the solutions of the eigenvalue problem with respect to the total Hamiltonian $H$ projected onto the subspace $P$ spanned by the Gaussian basis. The variational expansion is given by

$$
\Phi=\sum_{c} \sum_{i, j, k} c_{i, j, k}^{c}\left[\varphi_{i}^{c}\left(\mathbf{r}_{1}^{c}\right) \varphi_{j}^{c}\left(\mathbf{r}_{2}^{c}\right) \varphi_{k}^{c}\left(\mathbf{r}_{3}^{c}\right)\right]_{J, M}^{s y m m / a s y m}
$$

where $\varphi_{i}^{c}\left(\mathbf{r}_{\mu}^{c}\right)$ are the basis functions for the Jacobi coordinate $\mathbf{r}_{\mu}^{c}, \mu=1,2,3$, in the channel $c$. The Schrödinger equation takes form

$$
H_{P} \Phi_{v}=E_{v} \Phi_{v}
$$

and is solved as the matrix eigenvalue problem.

The basis functions $\varphi_{i}^{c}$ in (2) are either primitive Gaussians or the atomic orbitals formed out of these Gaussians (these two sets are connected via an unitary transformation). The sum over channels $c \equiv C ; l_{1}, l_{2}, l_{3}$ runs over the various Jacobi sets of coordinates (we call them rearrangement channels and number them with $C$, see Fig. 1) and various associated triple configurations of the angular momenta $l_{1}^{c}, l_{2}^{c}, l_{3}^{c}$ compatible with the coupling to the total angular momentum $J$.

The bracket around the triple products in (2) means that they are coupled to the total angular momenta $J, M$ and appropriately symmetrized. In the present paper we consider the $J=0^{+}$symmetry, i.e. the states with the total (orbital) angular momentum 0 and (natural) parity +1 , with the positrons in the singlet (spin 0 ) configuration. The spin is not treated explicitly, rather we symmetrize the spacial wave functions to be space symmetric with respect to the permutation of the spacial coordinates of the two positrons. The latter symmetrization is achieved by "doubling" the Jacobi rearrangement channels, i.e. by including their companions obtained by permutation $\mathcal{P}$ of the two positrons (except for those arrangements that contain the $e_{1}^{+}-e_{2}^{+}$coordinate and need not be symmetrized, since $\left.\varphi\left(\mathbf{r}_{e_{1}^{+}, e_{2}^{+}}^{c}\right) \stackrel{\mathcal{P}}{\longrightarrow}(-1)^{l_{c}} \varphi\left(\mathbf{r}_{e_{2}^{+}, e_{1}^{+}}^{c}\right)\right)$.

There are alltogether 15 Jacobi rearrangement channels for the $\bar{H} P s$ system. Our studies of the convergence of binding energy revealed that the most important ones are channels $13,1,10$ and 15 illustrated on Fig. 1 . The channel numbers $C=13,1,10,15$ conform to the previous literature [13]. Thus we use 7 arrangements: arrangements 13, 1,10; their companions obtained by permutation of the two positrons; and arrangement 15 (which contains the $e_{1}^{+}-e_{2}^{+}$coordinate and does not need symmetrization).

Our calculations show that the most important contribution to the binding energy comes from the molecular $\bar{H}-P s$ channel $(C=13)$. Next in importance is the atomic channel with the electron orbiting the $\bar{H}^{+}$atomic ion core $(C=1)$. Next comes the positronium ion channel, $P s^{+}+\bar{p}(C=10)$. After that comes the repulsion (or total break up) channel where the $\mathrm{CM}$ of two mutually repelling positive particles is connected to the CM of two mutually repelling negative particles $(C=15)$.

The convergence of the binding energy is shown in Table 1 and illustrated on Fig. 2. The achieved accuracy is on the order of few micro-Hartrees. 
Table 1 Binding energy of the ground state of $\bar{H} P s$ against the number of basis functions $N_{\text {tot }}$ and against the number of channels $n_{\mathrm{ch}}^{\text {tot }}$

\begin{tabular}{lllll}
\hline$N_{\text {tot }}$ & $n_{\text {ch }}^{\text {tot }}$ & c.f. Fig. 2 & $E$ & $C-l \lambda L$ \\
\hline 3375 & 1 & a & -0.7745429 & $13-000$ \\
6750 & 2 & b & -0.7826201 & $+1-000$ \\
10125 & 3 & c & -0.7847746 & $+10-000$ \\
13500 & 4 & d & -0.7868436 & $+15-000$ \\
14500 & 5 & e & -0.7878861 & $+13-110$ \\
15500 & 6 & f & -0.7884728 & $+13-011$ \\
16500 & 7 & & -0.7885554 & $+13-101$ \\
17500 & 8 & & -0.7885777 & $+1-110$ \\
18500 & 9 & & -0.7885947 & $+1-101$ \\
19500 & 10 & $\mathrm{~g}$ & -0.7887261 & $+10-110$ \\
20500 & 11 & & -0.7887341 & $+10-011$ \\
21500 & 12 & & -0.7887643 & $+10-101$ \\
22500 & 13 & h & -0.7888232 & $+15-011$ \\
28132 & 24 & $\mathrm{i}$ & -0.7888509 & $+13 / 1 / 10 / 15-(220,022,202)$ \\
32740 & 33 & $\mathrm{j}$ & -0.7888658 & $+13 / 1 / 10 / 15-(211,112,121)$ \\
35827 & 42 & $\mathrm{k}$ & -0.7888665 & $+13 / 1 / 10 / 15-(330,033,303)$ \\
41658 & 59 & 1 & -0.7888674 & $+13 / 1 / 10 / 15-(123,132,213,231,312,321)$ \\
45431 & 70 & $\mathrm{~m}$ & -0.7888675 & $+13 / 1 / 10 / 15-(440,044,404)$ \\
Ref. [8] Bubin and Varga & -0.7888707 & \\
\hline
\end{tabular}

Last column shows which channels are included. The notation $+13 / 1 / 10 / 15-(220,022,202)$ means that we added the essential combinations with $l \lambda L=220,022,202$ for channels $13,1,10$ and 15 . The adopted proton mass is $m_{\mathrm{p}}=1836.15267247$

\section{Calculations of the scattering length and the scattering cross sections for antihydrogen - positronium collisions}

The outer part of the total wave function $\Psi$ describes the asymptotic behavior of the system (cf. (1)). It is a sum over channel functions that satisfy the correct scattering boundary conditions. The sum runs over open physical channels $c$, i.e. over various possible fragmentations of the system and the angular momenta of the relative motion of the fragments. We include both the so called $H$ channels (fragmentation into two two-body fragments) and $K$ channels (fragmentation into a three-body fragment and a single particle). Hence $\phi_{c}^{(2)}, \phi_{c}^{(3)}$ in (1) are the wave functions of isolated 2 and 3 body fragments in channel $c ; \mathbf{r}_{c}$ and $\mathbf{q}_{c}$ are the internal coordinates of these fragments.

The relative motion of the fragments in various scattering channels is given by the functions $\chi_{c}\left(R_{c}\right)$, with $\mathbf{R}_{c}$ being the Jacobi coordinate for this motion. If these fragments are neutral (e.g. the atomic fragments) the wave functions of relative motion satisfy

$$
\lim _{R_{c} \rightarrow \infty} \chi_{c}\left(R_{c}\right)=u_{l}^{(-)}\left(k_{c} R_{c}\right) \delta_{c c_{i}}-\sqrt{\frac{v_{c_{i}}}{v_{c}}} S_{c, c_{i}} u_{l}^{(+)}\left(k_{c} R_{c}\right),
$$




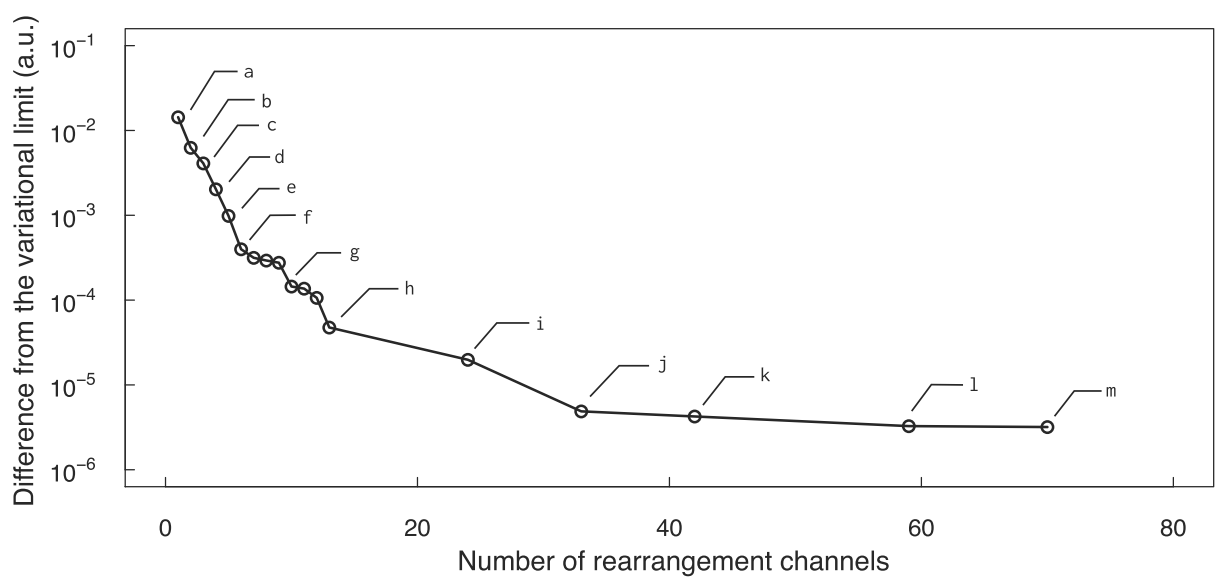

Fig. 2 Convergence of the binding energy as a function of the number of channels $c=(C ; l \lambda L)$ included in the calculation. The channels corresponding to each point and the concomitant size of the Gaussian basis are specified in Table 1. Our experience shows that it is more important to increase the number of channels than to merely increase the number of the Gaussian functions spanning each channel

with $u_{l}^{( \pm)}\left(k_{c} R_{c}\right)=R_{c} h_{l}^{( \pm)}\left(k_{c} R_{c}\right)$ being the Ricatti-Hankel functions [17], $v_{c_{i}}, v_{c}$ being the velocities of the fragments in the initial and final channel, respectively, and $S_{c, c_{i}}$ being the elements of the scattering $S$-matrix.

If the fragments are charged (as in partitioning into a 3-body system and a lone particle), the function of the relative motion satisfies

$$
\lim _{R_{c} \rightarrow \infty} \chi_{c}\left(R_{c}\right)=-\sqrt{\frac{v_{c_{i}}}{v_{c}}} S_{c, c_{i}} u_{l}^{(C+)}\left(k_{c} R_{c}\right),
$$

where $u_{l}^{(C+)}\left(k_{c} R_{c}\right)$ is the outgoing spherical Coulomb function [18].

Actually, the $S$-matrix elements in the above equations depend not only on the initial and final angular momenta given by $c_{i}$ and $c$, but also on the total angular momentum of the system $J$, its parity, and the intermediate couplings of the angular momenta of the collision fragments. More about this is coming later in the text.

It is important to emphasize that functions $\chi_{c}\left(R_{c}\right)$ are not requested to vanish in the inner region (except for $R_{c}=0$ ). In that way they can contribute to the description of the inner region spanned by functions $\Phi_{v}$, and at the same time facilitate a smooth transition between the inner and outer regions.

Solving the Schrödinger equation (3) with $\Psi$ expressed as in (1) becomes equivalent to the determination of expansion coefficients $b_{v}$ and the channel functions $\chi_{c}$. The strategy has been discussed in ref. [19] for the case of $\bar{H} H$. The solution of the eigenvalue problem is converted into the solution of the system of non-local, coupled integro-differential equations. We show the general form of these equations in the Appendix, postponing the derivations and description of numerical procedures to the more comprehensive publication. The objective is then to solve these equations as to simultaneously and self-consistently determine the outer part $\left(\chi_{c}\right.$ and thereby $\left.S_{c, c^{\prime}}\right)$ and the inner part $\left(b_{v}\right)$. The asymptotic functions $\chi_{c}$ are determined by numerical integration using the Compact Finite Difference Method (CFDM) that takes into account the boundary conditions.

The expansion coefficients $b_{v}$ can be expressed in terms of channel functions $\chi_{c}$ [19]. This suggests the use of iterative procedure. However, the attempts to solve the problem 
Table 2 Notation for scattering channels

\begin{tabular}{lll}
\hline Channel state & Fragments & Description \\
\hline$\alpha_{1}$ & $\bar{H}(1 s)+P s(1 s)$ & elastic scattering \\
$\alpha_{2}$ & $\bar{H}(1 s)+P s(2 s)$ & excitation of $P s(2 s)$ \\
$\alpha_{3}$ & $\bar{H}(1 s)+P s(2 p)$ & excitation of $P s(2 p)$ \\
$\alpha_{4}$ & $\bar{H}(1 s)+P s(3 s)$ & excitation of $P s(3 s)$ \\
$\alpha_{5}$ & $\bar{H}(1 s)+P s(3 p)$ & excitation of $P s(3 p)$ \\
$\alpha_{6}$ & $\bar{H}(1 s)+P s(3 d)$ & excitation of $P s(3 d)$ \\
$\alpha_{7}$ & $\bar{H}+e$ & production of $\bar{H}^{+}$ \\
\hline
\end{tabular}

iteratively did not succeed, we were not able to achieve convergence. Instead, we have solved the problem by casting the numerical integration into algebraic equations which were solved as the matrix problem, determining the values of the functions $\chi_{c}$ on the grid, in form of one vector for all functions $\chi_{c}$. Subsequently, the scattering matrix elements $S_{c, c_{i}}$, and the cross sections are determined, i.e. $\chi_{c} \longrightarrow S_{c, c_{i}} \longrightarrow \sigma_{c, c_{i}}$.

To construct the cross sections we need a more subtle notation for the channel angular momenta. We replace the symbol $c$ by a pair $\alpha, \lambda$ where $\alpha$ defines the angular momenta of the collisional fragments $l$ and $L$ (see Table 2 and Fig. 1), and $\lambda$ is the angular momentum of their relative motion. We also need to take into account that the three angular momenta that characterize the channels $c$ can be coupled in different ways. We adopt the coupling scheme where the momenta of the scattering fragments $(l$ and $L)$ in the channel state $\alpha$ are added first and the result $(\Lambda)$ is added to the momentum of the relative motion of the fragments $\lambda$, to give the total angular momentum $J$.

The cross sections, for scattering from the initial state $\alpha_{i}$ in the partial wave $\lambda_{i}$ into a final state state $\alpha$ in a partial wave $\lambda_{f}$, are given by

$$
\sigma_{\alpha, \alpha_{i}}^{\lambda_{f}, \lambda_{i}}\left(J, \Lambda_{i}, \Lambda_{f} ; E\right)=\frac{\pi}{k_{\alpha_{i}}^{2}}\left(2 \lambda_{i}+1\right)\left|\delta_{\alpha, \alpha_{i}}-S_{\alpha, \alpha_{i}}^{\lambda_{f}, \lambda_{i}}\left(J, \Lambda_{i}, \Lambda_{f} ; E\right)\right|^{2} .
$$

The above partial cross sections need to be calculated for each $\lambda_{i}=0,1,2 \ldots$, for the associated values of $\left|\lambda_{i}-\Lambda_{i}\right| \leq J \leq\left(\lambda_{i}+\Lambda_{i}\right)$ and for the allowed values of $\lambda_{f}$, compatible with $J$ and $\Lambda_{f}$. The above partial cross sections can be summed up to give the cross section for scattering from the initial state $\alpha_{i}$ to a final state $\alpha$

$$
\sigma_{\alpha, \alpha_{i}}(E)=\sum_{J} \sum_{\Lambda_{i}, \Lambda_{f}} \sum_{\lambda_{i}, \lambda_{f}} \sigma_{\alpha, \alpha_{i}}^{\lambda_{f}, \lambda_{i}}\left(J, \Lambda_{i}, \Lambda_{f} ; E\right) .
$$

Finally, the state to state cross sections can be summed up over the final states to give the total cross section

$$
\sigma_{t o t, \alpha_{i}}(E)=\sum_{\alpha} \sigma_{\alpha, \alpha_{i}}(E) \theta\left(E-E_{\alpha}\right)
$$

where $\theta$ is the Heaviside step function and $E_{\alpha}$ is the collision energy threshold above which the reaction to state $\alpha$ becomes open.

In the present work we consider the scattering of $\bar{H}(1 s)$ and $P s(1 s)$ below the $\bar{H}(n=2)$ threshold and for this case the notation in (6) - (8) becomes significantly simpler, because 


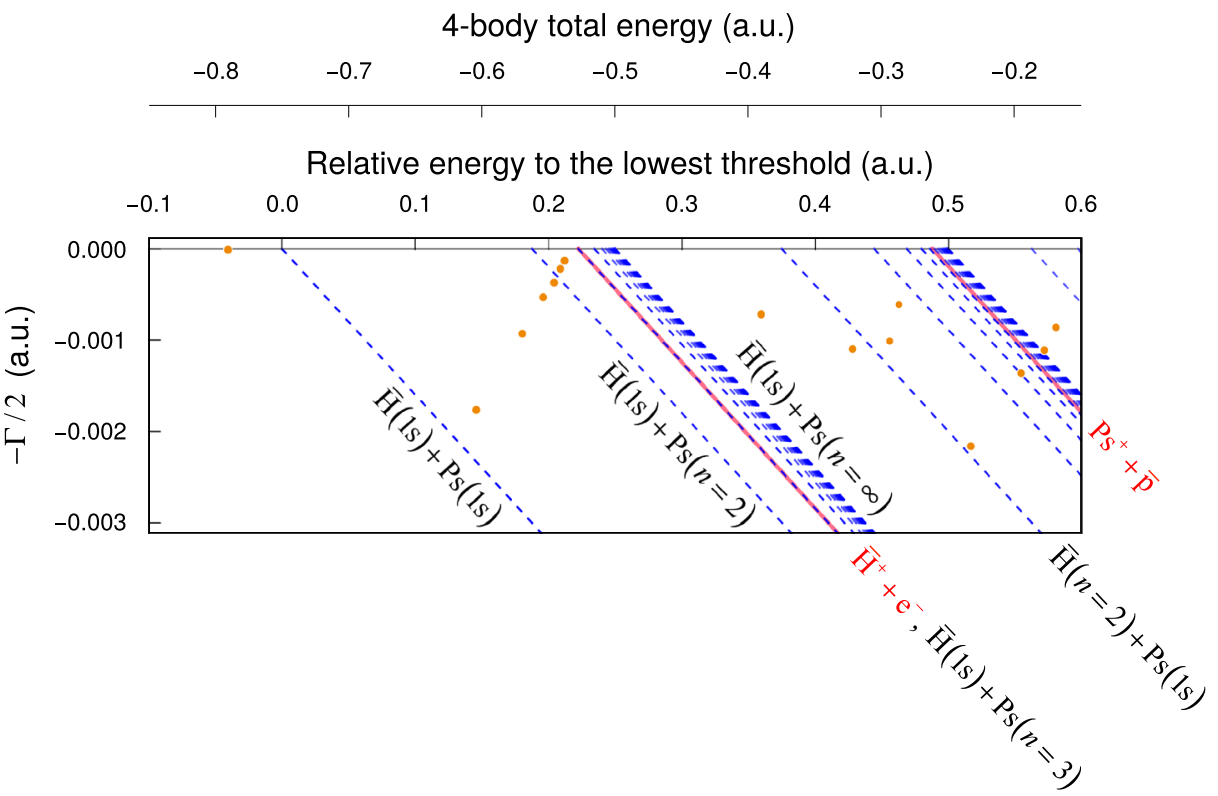

Fig. 3 The energy spectrum of the $\bar{H} P s$ system of the $J=0^{+}$symmetry with positrons in the singlet (spin 0) configuration. There are two energy scales: the total 4-body energy, and the energy relative to the lowest threshold. The orange dots indicate the resonances. There exists only one bound state, just below the threshold for the dissociation into antihydrogen and positronium atoms in their ground states. The various branches of the continuous spectrum are indicated by blue lines, originating at the thresholds. We see the thresholds for positronium excitation, which accumulate at the 3-body fragmentation threshold

$\Lambda_{i}=l_{i}=L_{i}=0, J=\lambda_{i}$ and $\Lambda_{f}=L_{f}$. Therefore, for this case the equation (7) becomes

$$
\sigma_{\alpha, \alpha_{i}}(E)=\sum_{J} \sum_{\lambda_{f}=\left|J-L_{f}\right|}^{\lambda_{f}=J+L_{f}} \sigma_{\alpha, \alpha_{i}}^{\lambda_{f}, \lambda_{i}}(J ; E) .
$$

In the above equation, $J$ is equivalent to the relative angular momentum in the $\bar{H}(1 s)+$ $\operatorname{Ps}(1 s)$ scattering $\left(J=\lambda_{i}\right)$ and thus $\sigma_{\alpha, \alpha_{i}}^{\lambda_{f}, \lambda_{i}}\left(J=\lambda_{i} ; E\right)$ can be regarded as a partial cross section for scattering from the initial state $\alpha_{i}=\alpha_{1}$ in $J$-wave to the final state $\alpha$ in $\lambda_{f}$-wave.

In the following we present the cross sections for the $s$-wave scattering between $\bar{H}(1 s)$ and $P s(1 s)$, thus setting $\alpha_{i}=\alpha_{1}$ (see Table 2) and $\lambda_{i}=0$. This situation conforms entirely to the $J=0$ symmetry (the wave functions possess the $J=0^{+}$, singlet positronic configuration symmetry). The excitation of the positronium states $P s\left(n_{f}, L_{f}\right)$ is possible but only in the final partial waves with the angular momenta satisfying $\lambda_{f}=L_{f}$.

The considered range of collisional energies exceeds the threshold for $\bar{H}^{+}$formation. This means that we need to solve the coupled channel problem for all seven opened channels, and determine the $7 \times 7$ scattering matrix S. The energy spectrum of $\bar{H} P$ s showing the scattering channels is shown on Fig. 3. The channel labeling for the included coupled channels is given in Table 2. (Please note that the number of open scattering channels only accidentally coincides (and should not be confused) with the 7 arrangement channels included in the calculation of the inner part of the wave function.)

The results for the $s$-wave elastic scattering from the one channel calculation are presented in Fig. 4. 


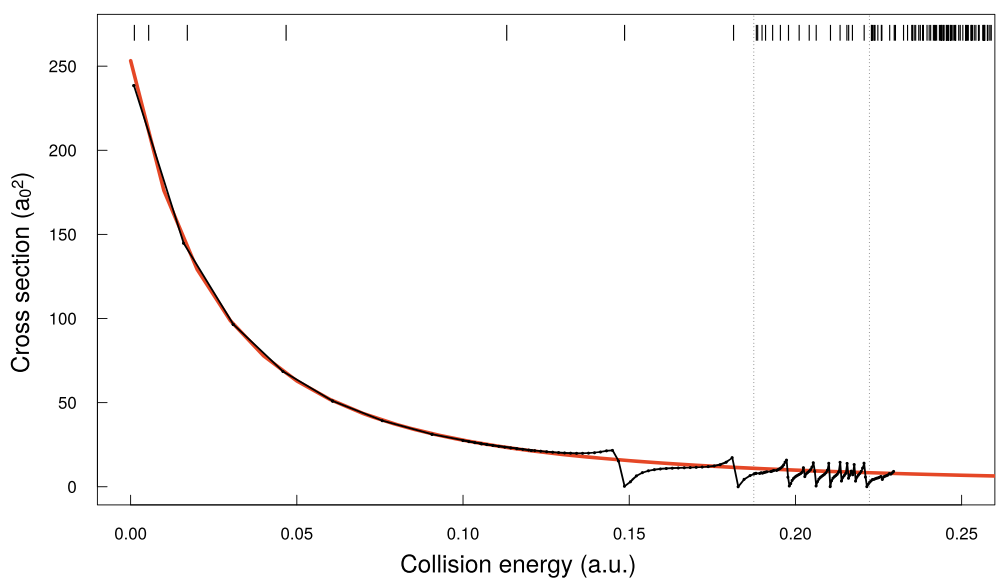

Fig. 4 Elastic cross section $\sigma_{1,1}^{0,0}(E)$. Black: CRC, one channel. Red: PM/ERT. The vertical dotted lines indicate the thresholds for $P s(2)$ and $P s(3)$ excitations. The bars along the upper edge indicate the eigenvalues of the 4-body eigenvalue problem for the inner part

The one channel calculation is exact below the first excitation threshold for $\operatorname{Ps}(n=2)$. The construction of the inner part of the scattering wave function is similar to that for the bound state calculation (shown in Table 1). The latter was constructed using 41648 fourbody basis functions from 16 channels in 7 sets of Jacobi coordinates, including angular momentum configurations with $l_{\mu}^{c} \leq 2$. In the scattering calculations we use the similar size expansion (44 496 basis functions) to describe the closed part of the wave function (the first summation in (1)). However the number of channels is reduced, so that the closed part can be considered to be a restriction of the one used in the calculation of the binding energy of $\bar{H} P$ s. On the other hand we have extended the size of the manifolds along the coordinates describing the relative motion of the scattering fragments, especially by additional longrange functions along the $\bar{H}^{+}-e$ coordinate as to describe the expected oscillation structure of the resonant states.

The basis functions for the 2-body (atomic) and 3-body (ionic) fragments (appearing in the second and third summation in (1)) are obtained by separate 2 and 3 body calculations. These functions are compact but of good quality. Thus for instance the fragment of antihydrogen ion $\bar{H}^{+}$is expanded in 736 basis functions, resulting in the binding energy 0.527442 a.u. (and thus differing from the best available reference value only by 4 micro Hartrees).

Suma sumarum, at the present stage of calculations, our scattering expansions are less elaborate than the one for the bound state. This is why the accuracy of the binding energy of $\bar{H} P s$ is on the order of $5 \mathrm{ppm}$ (parts per million) whereas the non-unitarity of the scattering matrix for the here reported scattering calculations is on the order of $5 \%$ (at worst). ${ }^{1}$ Still, the quality of the 4-body inner part is such that already the one channel calculation is able to discern the resonant features both below and above the first excitation threshold, and at the same time give the very competitive scattering length.

\footnotetext{
${ }^{1}$ Note added in proof: In the next generation of calculations, with more experience we have been able to reduce this non-unitarity to $2.6 \%$ at worst, without extending the computational effort.
} 


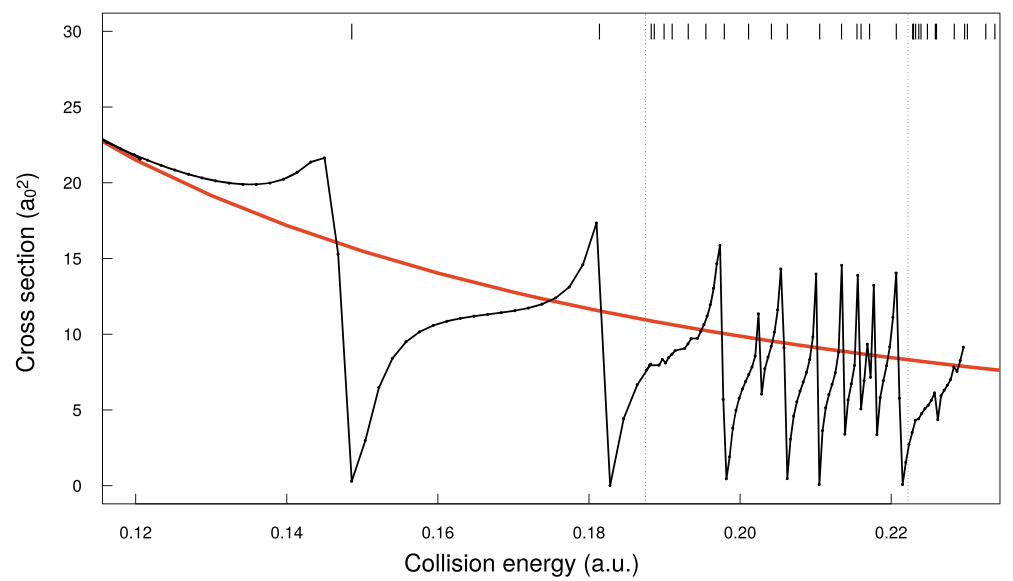

Fig. 5 Elastic cross section, close-up through the resonance region. Black: CRC. Red: PM/ERT. The simple PM/ERT method gives remarkably good overall elastic cross section, even above the threshold for $P s$ excitation, but fails to reproduce the resonant features

Table 3 Scattering length and elastic scattering at $E=0$

\begin{tabular}{lll}
\hline Scattering length $a\left[a_{0}\right]$ & $\sigma_{11}^{0,0}(E=0)\left[a_{0}^{2}\right]$ & source \\
\hline $4.488 a_{0}$ & $253.11 a_{0}^{2}$ & This work simple Projection Method (PM/ERT) \\
$4.410 a_{0}$ & $244.39 a_{0}^{2}$ & Blackwood et al. [10] coupled pseudostates / R-matrix \\
$4.340 a_{0}$ & $236.69 a_{0}^{2}$ & Ivanov et al. [6] Stochastic Variational Method (SVM ) \\
$4.331 a_{0}$ & $235.71 a_{0}^{2}$ & Woods et al. [12] complex Kohn variational method \\
$4.291 a_{0}$ & $231.38 a_{0}^{2}$ & This work GEM/CRC calculation \\
\hline
\end{tabular}

We mention in passing that the good quality of the inner part of the scattering wave function means that it alone can be used to get the first estimation of the elastic cross section. This is shown on Fig. 4 and in Table 3 which include the results for the elastic cross section obtained by the simple Projection Method combined with the Effective Range Theory (PM/ERT) [20]. The importance of the good description of the inner part (reflected e.g. in the resulting binding energy) on the scattering length has been noted before, e.g. in ref. [10].

To judge the accuracy of the obtained cross sections at low energies we report the scattering length $a$, related to the low energy limit of the elastic scattering according to $\sigma_{11}^{0,0}(E \rightarrow 0)=4 \pi a^{2}$. Comparison of our scattering length to other calculations is given in Table 3.

Since the scattering length obtained from a variational calculation is tacitly assumed to be an upper bound for this quantity, our result is seen to be highly accurate. The accuracy of the cross sections at higher energies is estimated from the residual non-unitarity of the scattering matrix $\mathrm{S}(E)$.

On Fig. 6 we illustrate the importance of channel coupling and the influence of inelastic scattering on the elastic one. Even though the elastic scattering dominates, the inelastic one has a substantial effect. We see that the pure one-channel elastic scattering (black broken 


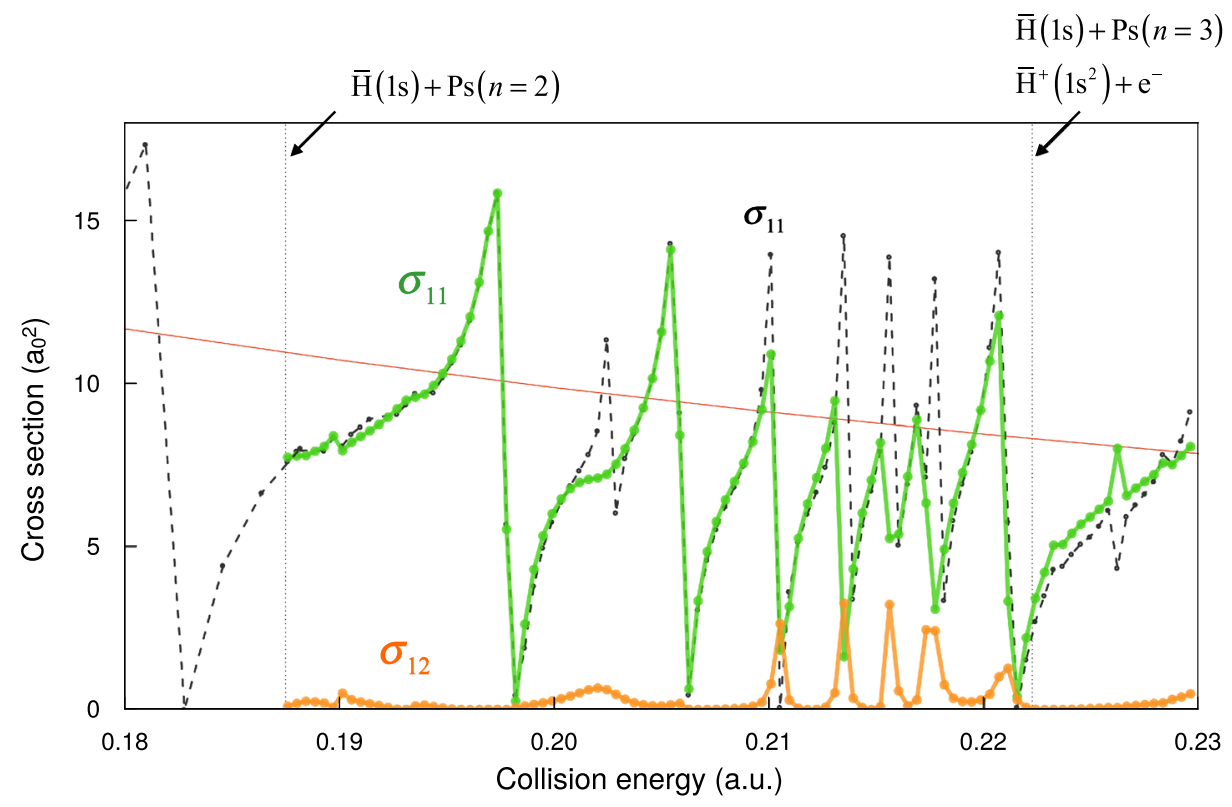

Fig. 6 Elastic and inelastic scattering above the lowest excitation threshold and across the resonant region. Red line: ERT. Broken line: CRC, elastic scattering $\sigma_{11}^{0,0}$, one channel calculation. Green line: CRC, elastic scattering $\sigma_{11}^{0,0}$ in the presence of inelastic scattering, 2 channel calculation. Orange line: CRC, cross section for inelastic (excitation) scattering $\sigma_{21}^{0,0}$ in the presence of elastic scattering, 2 channels calculation

line) is reduced in magnitude and its resonant features are remodulated in the presence of inelastic scattering (two-channel calculation, green line).

On Fig. 7 we show the cumulative scattering cross sections resulting from the 3 -channels calculation, i.e. the sum of $\sigma_{11}^{0,0}, \sigma_{21}^{0,0}$ and $\sigma_{31}^{1,0}$. The sum of these 3 cross sections does not correspond to the pure partial wave scattering. Rather, we include those partial waves that are compatible with the total angular momentum $J=0$ for the entire system, since at the moment we consider collisions of $\bar{H}(1 s)$ with $P s(1 s)$ with $\lambda_{i}=0$ for the initial relative motion (low energy collisions). Hence, the outcoming products $\bar{H}(1 s)$ and $P s(2 p)$ are kept in the $\lambda_{f}=1$ partial wave as to ensure conservation of the total angular momentum.

The three different shades of Fig. 7 show the separate contributions and the cumulative effect of the presented cross sections. Each of them is obtained from the common coupled 3channels calculation. We notice that $\sigma_{21}^{0,0}, \sigma_{31}^{1,0}$ start to contribute above the lowest inelastic channel (that of $\operatorname{Ps}(n=2)$ excitation) and that the behavior of the cross sections across the thresholds for $P s(n=2)$ and $P s(n=3)$ excitations is markedly affected by the nearby resonances.

To analyse the resonant structure of the cross section we have located the resonances through an independent calculation, using the complex coordinate method and solving the complex eigenvalue problem. As seen from the upper panel of Fig. 7 the complex eigenvalues that determine the positions and the life-times of the resonances correspond very well to the resonant features seen in scattering cross sections obtained by the CRC method.

The results of the 7-channels calculation, coupling the $\bar{H}^{+}+e$ channel and all open channels below, is presented on Fig. 8. In the energy interval shown in Fig. 8 the S-matrix 


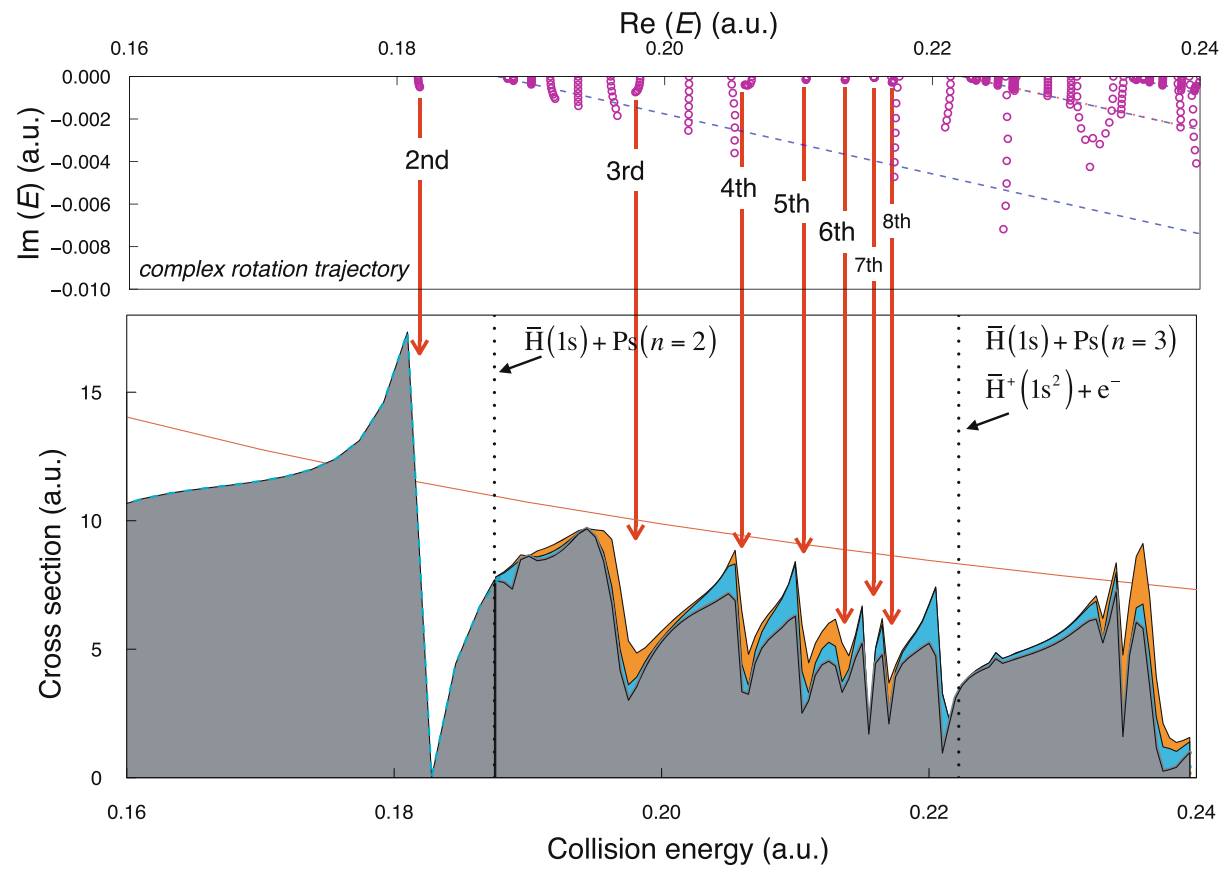

Fig. 7 Scattering cross section in the energy region between the first and second excitation thresholds, 3channels calculation. The contributions from various channels are indicated using different colors. Grey shade: elastic scattering. Blue shade: excitation of the $P s(2 s)$. Orange shade: excitation of the $P s(2 p)$. The red line indicates the elastic scattering obtained with the PM/ERT. The upper panel shows the complex eigenvalues corresponding to the resonances of $\bar{H} P s$, as they appear from the separate 4-body, complexcoordinate calculation

satisfies the unitarity condition with the accuracy of 5\% at worst. We notice that, once the $\bar{H}^{+}$production sets in, it becomes the strongest inelastic process with the cross section on the order of 1 a.u. .

\section{Summary}

We have presented the first round of the 4-body, Coupled Rearrangement Channels calculations for the $\bar{H} P s$ system. The simultaneous use of several sets of Jacobi coordinates in the same calculation allows the systematic analysis of the binding, structure and scattering properties of the system. It also alleviates the problem of linear dependencies (at the present level of the description we have not noticed it at all). Using the 7 most essential rearrangement channels we have achieved the binding energy of the ground state $E_{b}=0.7888675$ a.u. which indicates the accuracy on the order of micro-Hartrees.

We have then used the 4-body solutions of the eigenvalue problem for $\bar{H} P s$ as the basis for the expansion of the inner part of the total scattering wave function. The asymptotic behavior of that wave function, rendering the scattering matrix elements, was determined through solving the coupled, integro-differential equations. We have solved these equations including all open scattering channels for energies above the threshold for the rearrangement reaction $\bar{H}^{+}+e$. We have calculated the $s$-wave cross sections for the elastic scattering, the 


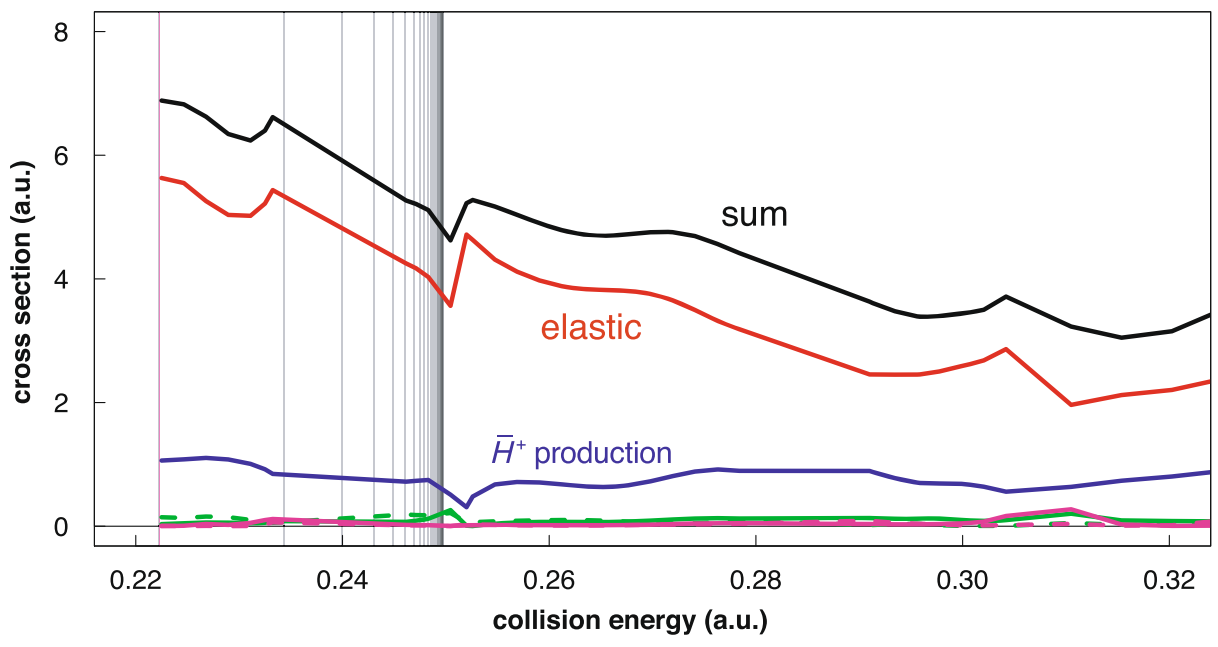

Fig. 8 Scattering cross section in the energy region above the threshold for $\bar{H}^{+}$production, 7-channels calculation. The vertical lines show the series of $H(1)+P s(n)$ excitation thresholds, starting from $H(1)+$ $P s(3)$ and converging to $H(1)+e^{+}+e$ three body fragmentation threshold. Red line: elastic scattering $\sigma_{11}^{0,0}$. Green, solid line: $\operatorname{Ps}(2 s)$ excitation $\sigma_{21}^{0,0}$; broken line: $P s(2 p)$ excitation $\sigma_{, 1}^{1,0}$. Mangenta, solid line: $P s(3 s)$ excitation $\sigma_{41}^{0,0}$; broken line: $\operatorname{Ps}(3 p)$ excitation $\sigma_{51}^{1,0}$; dotted line: $P s(3 d)$ excitation $\sigma_{61}^{2,0}$. Blue line: $\bar{H}^{+}$production $\sigma_{71}^{0,0}$. Black line: sum of the cross sections mentioned above

inelastic scattering ending with excitation of the positronium, and the rearrangement reaction ending with the $\bar{H}^{+}$ion. We have seen that, notwithstanding some resonant features, above the threshold for the $\bar{H}^{+}$production this reaction is the strongest inelastic process, with the cross section on the order of 1 a.u. The resonant features, attributed to the presence of charged fragments in the $\bar{H}^{+}+e$ channel, are seen already in the purely elastic scattering, but, as seen from our coupled-channel procedure, are then substantially modulated by the presence of inelastic processes.

The accuracy of our cross-section calculations can be inferred from the residual nonunitarity of the S-matrix. In the presented energy range of the scattering calculations, the unitarity of the S-matrix has been satisfied to within 5\% (at worst).

For the low energy scattering, the indication of the accuracy may be obtained from the consideration of the low energy limit of the elastic scattering, which becomes $\sigma_{11}^{0,0}(E=$ $0)=231.38 a_{0}^{2}$. The corresponding scattering length is $a=4.291 a_{0}$ which is currently the lowest informal upper bound for this quantity.

Acknowledgments We gratefully acknowledge the financial support from the Japan Society for the Promotion of Science (JSPS) and from the Swedish Research Council. T. Y. was financially supported by Grant-in-Aid for JSPS Research Fellow Grant Number JP16J02658 and JSPS Overseas Challenge Program for Young Researchers. Y. K. was supported by JSPS KAKENHI Grant Number JP17K05592.

\section{Appendix}

We display below the generic form of the coupled integro-differential equations for channel functions, to show the appearance of the non-local potentials and coupling of the rearrangement channels arising from the permutation of the two positrons. For simplicity and 
brevity we show the case of the elastic $\bar{H}-P s$ scattering. The case involving inelastic and rearrangement channels is a non straightforward generalization of the equations below.

The channel functions are solutions of the following equations:

$$
\begin{aligned}
& \left(\frac{\mathrm{d}^{2}}{\mathrm{~d} R_{\alpha}^{2}}-\frac{J(J+1)}{R_{\alpha}^{2}}-2 \mu_{\alpha} \tilde{V}_{\mathrm{int}}^{(\alpha)}\left(R_{\alpha}\right)+k_{\alpha}^{2}\right) \chi_{J}^{(J M)}\left(R_{\alpha}\right) \\
& +\eta\left(\frac{\mu_{\alpha}}{\mu_{\bar{\alpha}}}\right) \int R_{\alpha} R_{\bar{\alpha}} \mathrm{d} R_{\bar{\alpha}} W_{\mathrm{N}}^{(\alpha \bar{\alpha})}\left(R_{\alpha}, R_{\bar{\alpha}}\right)\left(\frac{\mathrm{d}^{2}}{\mathrm{~d} R_{\bar{\alpha}}^{2}}-\frac{J(J+1)}{R_{\bar{\alpha}}^{2}}+k_{\bar{\alpha}}^{2}\right) \chi_{J}^{(J M)}\left(R_{\bar{\alpha}}\right) \\
& +\eta\left(-2 \mu_{\alpha}\right) \int R_{\alpha} R_{\bar{\alpha}} \mathrm{d} R_{\bar{\alpha}} W_{\mathrm{V}}^{(\alpha \bar{\alpha})}\left(R_{\alpha}, R_{\bar{\alpha}}\right) \chi_{J}^{(J M)}\left(R_{\bar{\alpha}}\right) \\
& +\sum_{\gamma=\alpha, \bar{\alpha}} \eta_{\gamma}\left(-2 \mu_{\alpha}\right) \int R_{\alpha} R_{\gamma} \mathrm{d} R_{\gamma} W_{\mathrm{VV}}^{(\alpha \gamma)}\left(R_{\alpha}, R_{\gamma}\right) \chi_{J}^{(J M)}\left(R_{\gamma}\right)=0,
\end{aligned}
$$

where $k_{\alpha}^{2}=2 \mu_{\alpha}\left(E-\epsilon_{\overline{\mathrm{H}}(1 s)}-\epsilon_{\operatorname{Ps}(1 s)}\right), \alpha$ specifies a coordinate set (see Fig. 1) and $\bar{\alpha}$ specifies a coordinate set generated by permutation of two positrons; $\eta=1$ is used for the space-symmetric and $\eta=-1$ for the space-antisymmetric case, respectivly. In the following formulation, we use $\eta=1$. In the above we have introduced the following non-local potentials:

$$
\begin{aligned}
W_{\mathrm{N}}^{(\alpha \bar{\alpha})}\left(R_{\alpha}, R_{\bar{\alpha}}\right)= & \mathcal{J}_{\alpha \bar{\alpha}}\left\langle\phi_{\overline{\mathrm{H}}(1 s)}\left(r_{\alpha}\right) \phi_{\mathrm{Ps}(1 s)}\left(q_{\alpha}\right)\left[\left[Y_{0}\left(\hat{\mathbf{r}}_{\alpha}\right) \otimes Y_{0}\left(\hat{\mathbf{q}}_{\alpha}\right)\right]_{0} \otimes Y_{J}\left(\hat{\mathbf{R}}_{\alpha}\right)\right]_{J M}\right| \\
& \times 1\left|\phi_{\overline{\mathrm{H}}(1 s)}\left(r_{\bar{\alpha}}\right) \phi_{\operatorname{Ps}(1 s)}\left(q_{\bar{\alpha}}\right)\left[\left[Y_{0}\left(\hat{\mathbf{r}}_{\bar{\alpha}}\right) \otimes Y_{0}\left(\hat{\mathbf{q}}_{\bar{\alpha}}\right)\right]_{0} \otimes Y_{J}\left(\hat{\mathbf{R}}_{\bar{\alpha}}\right)\right]_{J M}\right\rangle_{\mathbf{q}_{\alpha}, \hat{\mathbf{R}}_{\bar{\alpha}}, \hat{\mathbf{R}}_{\alpha}},
\end{aligned}
$$

$$
\begin{aligned}
W_{\mathrm{V}}^{(\alpha \bar{\alpha})}\left(R_{\alpha}, R_{\bar{\alpha}}\right)= & \mathcal{J}_{\alpha \bar{\alpha}}\left\langle\phi_{\overline{\mathrm{H}}(1 s)}\left(r_{\alpha}\right) \phi_{\operatorname{Ps}(1 s)}\left(q_{\alpha}\right)\left[\left[Y_{0}\left(\hat{\mathbf{r}}_{\alpha}\right) \otimes Y_{0}\left(\hat{\mathbf{q}}_{\alpha}\right)\right]_{0} \otimes Y_{J}\left(\hat{\mathbf{R}}_{\alpha}\right)\right]_{J M}\right| \\
& \times V_{\text {int }}^{(\bar{\alpha})} \mid \phi_{\overline{\mathrm{H}}(1 s)}\left(r_{\bar{\alpha}}\right) \phi_{\operatorname{Ps}(1 s)}\left(q_{\bar{\alpha}}\right)\left[\left[Y_{0}\left(\hat{\mathbf{r}}_{\bar{\alpha}}\right) \otimes Y_{0}\left(\hat{\mathbf{q}}_{\bar{\alpha}}\right)\right]_{0} \otimes Y_{J}\left(\hat{\mathbf{R}}_{\bar{\alpha})}\right]_{J M}\right\rangle_{\mathbf{q}_{\alpha}, \hat{\mathbf{R}}_{\bar{\alpha}}, \hat{\mathbf{R}}_{\alpha}},
\end{aligned}
$$

where $\mathcal{J}_{\alpha \gamma}$ is the Jacobian of the coordinate transformation from the set $\left\{\mathbf{r}_{\alpha}, \mathbf{q}_{\alpha}, \mathbf{R}_{\alpha}\right\}$ to the set $\left\{\mathbf{q}_{\alpha}, \mathbf{R}_{\gamma}, \mathbf{R}_{\alpha}\right\}$, defined through the relationship d $\mathbf{r}_{\alpha} \mathrm{d} \mathbf{q}_{\alpha} \mathrm{d} \hat{\mathbf{R}}_{\alpha}=\mathcal{J}_{\alpha \gamma} \mathrm{d} \mathbf{q}_{\alpha} \mathrm{d} \mathbf{R}_{\gamma} \mathrm{d} \hat{\mathbf{R}}_{\alpha}$.

Furthermore,

$$
W_{\mathrm{VV}}^{(\alpha \gamma)}\left(R_{\alpha}, R_{\gamma}\right)=\sum_{v} V_{\alpha v}\left(R_{\alpha}\right) \frac{1}{E-E_{v}} V_{\gamma v}\left(R_{\gamma}\right)
$$

where $V_{\alpha v}\left(R_{\alpha}\right)$ is defined as

$$
\begin{aligned}
& V_{\alpha v}\left(R_{\alpha}\right)= \\
& \left\langle\phi_{\overline{\mathrm{H}}(1 s)}\left(r_{\alpha}\right) \phi_{\operatorname{Ps}(1 s)}\left(q_{\alpha}\right)\left[\left[Y_{0}\left(\hat{\mathbf{r}}_{\alpha}\right) \otimes Y_{0}\left(\hat{\mathbf{q}}_{\alpha}\right)\right]_{0} \otimes Y_{J}\left(\hat{\mathbf{R}}_{\alpha}\right)\right]_{J M}|(H-E)| \Phi_{v J M}\right\rangle_{\mathbf{r}_{\alpha}, \mathbf{q}_{\alpha}, \hat{\mathbf{R}}_{\alpha}} .
\end{aligned}
$$

There is also a local potential

$$
\begin{aligned}
\tilde{V}_{\text {int }}^{(\alpha)}\left(R_{\alpha}\right) & =\left\langle\phi_{\overline{\mathrm{H}}(1 s)}\left(r_{\alpha}\right) \phi_{\operatorname{Ps}(1 s)}\left(q_{\alpha}\right)\left[\left[Y_{0}\left(\hat{\mathbf{r}}_{\alpha}\right) \otimes Y_{0}\left(\hat{\mathbf{q}}_{\alpha}\right)\right]_{0} \otimes Y_{J}\left(\hat{\mathbf{R}}_{\alpha}\right)\right]_{J M}\right| \\
& \times V_{\text {int }}^{(\alpha)}\left|\phi_{\overline{\mathrm{H}}(1 s)}\left(r_{\alpha}\right) \phi_{\operatorname{Ps}(1 s)}\left(q_{\alpha}\right)\left[\left[Y_{0}\left(\hat{\mathbf{r}}_{\alpha}\right) \otimes Y_{0}\left(\hat{\mathbf{q}}_{\alpha}\right)\right]_{0} \otimes Y_{J}\left(\hat{\mathbf{R}}_{\alpha}\right)\right]_{J M}\right\rangle_{\mathbf{r}_{\alpha}, \mathbf{q}_{\alpha}, \hat{\mathbf{R}}_{\alpha}}
\end{aligned}
$$


with the interaction $V_{\text {int }}^{(\alpha)}$ defined by the relations

$$
\begin{aligned}
H & =T\left(\mathbf{R}_{\alpha}\right)+V_{\mathrm{int}}^{(\alpha)}+h_{\overline{\mathrm{H}}}+h_{\mathrm{Ps}}, \\
& =T\left(\mathbf{R}_{\bar{\alpha}}\right)+V_{\mathrm{int}}^{(\bar{\alpha})}+h_{\overline{\mathrm{H}}}+h_{\mathrm{Ps}},
\end{aligned}
$$

where $T\left(\mathbf{R}_{\alpha}\right)$ is the operator for the kinetic energy of relative motion along $\mathbf{R}_{\alpha}, h_{\bar{H}}$ the Hamiltonian for $\bar{H}, h_{\mathrm{Ps}}$ the Hamiltonian for $P s$, so that $V_{\text {int }}$ is the Coulomb interaction between $\bar{H}$ and $P s$.

The appearance of non-local potentials in the master equation (10) is of uttermost importance, since e.g. for the elastic scattering the local potential $\tilde{V}_{\text {int }}^{(\alpha)}\left(R_{\alpha}\right)(15)$ describing the effective $\bar{H}-P s$ interaction vanishes, due to the particular symmetry of $P s$, whose center of mass coincides with its geometrical center.

The above master equation (10) is solved using the compact finite difference method (CFDM) [19, 21]. The solution $\chi_{J}^{(J M)}$ is calculated as a vector on the grid

$$
\mathbf{v}=\left\{\chi_{J}^{(J M)}\left(r_{1}\right), \chi_{J}^{(J M)}\left(r_{2}\right), \cdots \chi_{J}^{(J M)}\left(r_{k}\right), \cdots \chi_{J}^{(J M)}\left(r_{N}\right)\right\}
$$

where $r_{1}, r_{2}, \cdots, r_{N}$ are the non-uniformly distributed grid points extending from the short range to the long range that in our calculations was typically outstretched to more than 100 a.u..

Open Access This article is distributed under the terms of the Creative Commons Attribution 4.0 International License (http://creativecommons.org/licenses/by/4.0/), which permits unrestricted use, distribution, and reproduction in any medium, provided you give appropriate credit to the original author(s) and the source, provide a link to the Creative Commons license, and indicate if changes were made.

\section{References}

1. Perez, P., Rosowsky, A.: A new path toward gravity experiments with antihydrogen. Nucl. Instr. Meth. Phys. Res. A 545, 20 (2005)

2. Chardin, G. et al.: GBAR, Proposal to measure the Gravitational Behaviour of Antihydrogen at Rest, CERN-SPSC-P-342, 30/09/2011. Technical report (2011)

3. Indelicato, P. et al.: The GBAR project, or how does antimatter fall? Hyp. Int. 228, 141 (2014)

4. Perez, P. et al.: The GBAR antimatter gravity experiment. Hyp. Int. 233, 21 (2015)

5. Yan, Z.-C., Ho, Y.K.: Ground state and S-wave autodissociating resonant states of positronium hydride. Phys. Rev. A 59, 2697 (1999)

6. Ivanov, I.A., Mitroy, J., Varga, K.: Positronium-hydrogen scattering using the stochastic variational method. Phys. Rev. A 65, 032703 (2002)

7. Mitroy, J.: Energy and expectation values of the PsH system. Phys. Rev. A 73, 054502 (2006)

8. Bubin, S., Varga, K.: Ground-state energy and relativistic corrections for positronium hydride. Phys. Rev. A 84, 012509 (2011)

9. Biswas, P.K.: Effect of $\mathrm{H}^{-}$ion formation on positronium-hydrogen elastic scattering. J. Phys. B 34, 4831 (2001)

10. Blackwood, J.E., McAlinden, M.T., Walters, H.R.J.: Positronium scattering by atomic hydrogen with inclusion of target excitation channels. Phys. Rev. A 65, 032517 (2002)

11. Comini, P. et al:: $\overline{\mathrm{H}}^{+}$production from collisions between positronium and $\mathrm{keV}$ antiprotons for GBAR. Hyp. Int. 228, 159 (2014)

12. Woods, D., Ward, S.J., Van Reeth, P.: Detailed investigation of low-energy positronium-hydrogen scattering. Phys. Rev. A 92, 022713 (2015)

13. Hiyama, E., Kino, Y., Kamimura, M.: Gaussian expansion method for few-body systems. Prog. Theor. Exp. Phys. 51, 223 (2003)

14. Hiyama, E.: Gaussian expansion method for few-body systems and its applications to atomic and nuclear physics. Progr. Theor. Exp. Phys. 01A204, 1-36 (2012) 
15. Kamimura, M.: Nonadiabatic coupled-rearrangement-channel approach to muonic molecules. Phys. Rev. A 38, 621 (1988)

16. Kino, Y., Kamimura, M.: Non-adiabatic calculation of muonic atom-nucleus collisions. Hyp. Int. 82, 45 (1993)

17. Taylor, J.R.: Scattering Theory. Wiley, Hoboken (1972)

18. Messiah, A.: Quantum Mechanics. North-Holland (1970)

19. Piszczatowski, K., Voronin, A., Froelich, P.: Nonadiabatic treatment of hydrogen-antihydrogen collisions. Phys. Rev. A 89, 062703 (2014)

20. Drachman, R.J., Houston, K.: Positronium-hydrogen elastic scattering. Phys. Rev. A 12, 885 (1975)

21. Zhao, J., Corless, R.M.: Compact finite difference method for integro-differential equations. App. Math. Comput. 177, 271 (2006)

Publisher's note Springer Nature remains neutral with regard to jurisdictional claims in published maps and institutional affiliations.

\section{Affiliations}

\section{Piotr Froelich ${ }^{1}$. Takuma Yamashita ${ }^{2}$ - Yasushi Kino ${ }^{2} \cdot$ Svante Jonsell $^{3}$. Emiko Hiyama ${ }^{4} \cdot$ Konrad Piszczatowski $^{1}$}

Takuma Yamashita

t.ymst@dc.tohoku.ac.jp

Yasushi Kino

y.k@m.tohoku.ac.jp

Svante Jonsell

jonsell@fysik.su.se

Emiko Hiyama

hiyama@riken.jp

Konrad Piszczatowski

konrad.piszczatowski@gmail.com

1 Department of Theoretical Chemistry, Uppsala University, 751 21, Uppsala, Sweden

2 Department of Chemistry, Tohoku University, Sendai, 980-8578, Japan

3 Department of Physics, Stockholm University, 106 91, Stockholm, Sweden

4 RIKEN, Nishina Center, Wako, 351-0198, Japan 